\title{
A Fully Nonlinear, Dynamically Consistent Numerical Model for Ship Maneuvering in a Seaway
}

\author{
Ray-Qing Lin ${ }^{1}$ and Weijia Kuang ${ }^{2}$ \\ ${ }^{1}$ Hydromechanics Department, David Taylor Model Basin, NSWCCD, 9500 MacArthur Boulevard, West Bethesda, \\ MD 20817-5700, USA \\ ${ }^{2}$ Planetary Geodynamics Laboratory, NASA Goddard Space Flight Center, Greenbelt, MD, USA
}

Correspondence should be addressed to Ray-Qing Lin, ray.lin@navy.mil

Received 28 March 2011; Accepted 19 July 2011

Academic Editor: Agostino Bruzzone

Copyright (c) 2011 R.-Q. Lin and W. Kuang. This is an open access article distributed under the Creative Commons Attribution License, which permits unrestricted use, distribution, and reproduction in any medium, provided the original work is properly cited.

This is the continuation of our research on development of a fully nonlinear, dynamically consistent, numerical ship motion model (DiSSEL). In this paper we report our results on modeling ship maneuvering in arbitrary seaway that is one of the most challenging and important problems in seakeeping. In our modeling, we developed an adaptive algorithm to maintain dynamical balances numerically as the encounter frequencies (the wave frequencies as measured on the ship) varying with the ship maneuvering state. The key of this new algorithm is to evaluate the encounter frequency variation differently in the physical domain and in the frequency domain, thus effectively eliminating possible numerical dynamical imbalances. We have tested this algorithm with several well-documented maneuvering experiments, and our results agree very well with experimental data. In particular, the numerical time series of roll and pitch motions and the numerical ship tracks (i.e., surge, sway, and yaw) are nearly identical to those of experiments.

\section{Introduction}

Ship motion with fixed heading is an overly simplification of general ship motion dynamics, for example, ship maneuvering in seaway is far more complex than the fixed heading. In our earlier work, Lin and Kuang [1] developed a fully nonlinear, dynamically consistent ship motion model (DiSSEL) that can accurately simulate ship motion with fixed heading. A continuing effort is therefore to expand DiSSEL for simulating ship motions when maneuvering in seaway.

Accurate prediction of ship motion when maneuvering in a seaway is one of the most challenging and important problems in ship motion dynamics. Much effort has been devoted in the past on modeling ship maneuvering in seaway. The earliest studies started from the simplest cases of simulation ship tracks (i.e., surge, sway, and yaw motions) in calm water. For example, Chaplin [2] and Folger et al. [3] predicted ship tracks in calm water based on empirical laws derived from experimental data. Söding [4] made a substantial step forward by including dynamics in ship motion modeling. He introduced a set of hydrodynamic maneuvering coefficients in simulation, though those coefficients are determined mainly from experimental data. Hooft and Pieffers [5] furthered this effort by including the effects of waves on ship motion (e.g., wave induced motions). In 1988, Lee [6] developed a potential flow method to calculate the rudder forces and the moments, thus making it possible to fully simulate ship maneuvering motion state. Nearly a decade late, Bailey et al. [7] proposed a hybrid approach in which experimental data and theoretical formulations (e.g., linearized or parameterized ship response function) are integrated into a single model, thus providing an enhanced description of fluid loading. More recently, several complicated computational models are developed for simulating complex cases, for example, the viscous flow models by Chau [8] and El Moctar [9].

These studies have greatly improved our understandings of ship tracks and ship maneuvering dynamics. The methodologies developed from these efforts are still widely used [1015]. However, as pointed by Söding [10], there are also many limitations in these methods. For example, the potential flow methods ignore the dissipative effects from (tiny) 
fluid viscosity, small-scale turbulence, and flow separation. The viscous flow models are capable of evaluating these dissipative effects. But they are computationally difficult and expensive: numerical instabilities and computational capability limitations make direct simulation in the oceanic parameter domain impossible. In addition, inclusion of any a priori condition (e.g., those empirical extrapolations from experimental data) will affect generality of the model.

To utilize the mathematical simplicity of potential flow approaches and to include appropriate dissipative effects, Lin and Kuang [16] developed a physically more realistic and computationally more efficient model in which the "blocking theory" is used to numerically determine the rudder forces, the lift forces, the moments, and the Kutta conditions at the trailing edges. Based on this approach, Lin et al. [17] were able to model accurately ship track and heel motion when maneuvering in calm water. Their results are well benchmarked by experiments data.

The natural extension of the effort by Lin and Kuang [16] and by Lin et al. [17] is to develop a dynamically consistent model for predicting ship maneuvering in seaway. The model by Lin and Kuang [1] can be a very good candidate for this purpose for several reasons: there is no a priori condition nor parameterization in this model, and the model is fully nonlinear and dynamically consistent. In particular, prediction of ship motion in fixed heading from this model agrees well with experiments. For predicting ship maneuvering in seaway, several mechanisms need to be included (or reintroduced) into this model, including change of ship heading, motion acceleration, and propeller forces.

But, one might anticipate a mathematical difficulty of modeling encounter waves in ship maneuvering. The wave frequency and the wave number vector of the encounter waves vary with the ship speed and ship heading. The variation due to the latter is in particular significant in ship maneuvering. As we will find later in this paper, changes in the encounter frequencies do have profound impacts on ship-motion interactions and thus on the ship motion state. In this paper, we will discuss the details of this model expansion, the mathematical/numerical difficulties, and the algorithms resolving these difficulties. This paper is organized as follows: in Section 2 is an overview of DiSSEL. Section 3 describes the details of the relevant algorithm. Model results validation is given in Section 4, followed by the discussions.

\section{Fundamentals of DiSSEL Nonlinear Ship Motion Model}

DiSSEL is a hybrid-flow ship motion model in time domain: the surface waves are described by velocity potential, but the fluid viscous effect is included in the form of wave breaking and wave blocking (separation effects) $[1,16,18]$. Inclusion of the viscous effect makes DiSSEL satisfying the Kutta condition at a trailing edge, and velocity stagnation points $(\mathbf{V}=0)$, thus allowing accurate evaluation of the lift force [17]. This hybrid-flow approach builds a solid foundation of our effort to expand the model for ship maneuvering in a seaway.

In DiSSEL, ship-wave interaction and ship solid body motion dynamics are modeled by two separate components.
Interactions between the two dynamical processes are determined through the pressure force on the ship hull surface and the restoring force from the water displaced by underwater ship volume. For the details of the partial differential equations, the boundary conditions and the surface integrations, we refer the reader to Lin et al. [19], Lin and Kuang $[1,20]$.

To expand DiSSEL for a ship maneuvering in a seaway, it is necessary to include a propulsion mechanism. As the first step, this mechanism can be approximated from experimental data (empirical). This approximation does not affect the dynamical consistency of our model, since the propulsion is determined by the ship engine, independent of the ship-wave interactions. In the following, we focus on the differences in the equations and the physical variables that are different from those of the original DiSSEL model.

Another notice should be made on the definitions of the reference frames used in DiSSEL. In Lin and Kuang [1], two additional reference frames are introduced: the ship reference frame (SRF) and the model reference frame (MRF). The latter is defined to be the reference frame moving horizontally with the ship mass center, but with the origin on the mean free surface. When a ship maneuvers in seaway, its track direction can change. But MRF does not change its orientation. Therefore, the rotational effect from this direction change needs to be accounted for correctly. For details of the reference frames, we refer the reader to Lin and Kuang [1].

2.1. Ship-Wave Interaction Model. In DiSSEL, the surface waves are described by the following set of partial differential equations:

$$
\begin{aligned}
& \nabla^{2} \varphi \equiv \nabla_{h}^{2} \varphi+\frac{\partial^{2} \varphi}{\partial^{2} z}=0, \quad(-H \leq z \leq \eta) \\
& \frac{\partial \varphi}{\partial t}+ {\left[\frac{1}{2} \nabla \varphi-\left(\mathbf{u}_{s}+\mathbf{v}_{h}\right)\right] \cdot \nabla \varphi+\frac{p}{\rho_{w}}+g z } \\
&-\frac{\partial\left(\mathbf{u}_{s}+\mathbf{v}_{h}\right)}{\partial t} \cdot \mathbf{x}-\nu \nabla_{h}^{2} \varphi=0, \quad(-H \leq z \leq \eta), \\
& \frac{\partial \eta}{\partial t}+\left(\nabla_{h} \eta\right) \cdot\left[\nabla_{h} \varphi-\left(\mathbf{u}_{s}+\mathbf{v}_{h}\right)\right]=\frac{\partial \varphi}{\partial z}, \quad(z=\eta),
\end{aligned}
$$

where $p$ is the pressure, $\rho_{w}$ is the water density, $v$ is the turbulent viscosity (arising from the wave-breaking processes), $\mathbf{x}$ is the position vector, $H$ is the water depth, $\varphi$ is the velocity potential, $\eta$ is the free surface elevation, $\nabla(\equiv$ $\hat{\mathbf{x}} \partial / \partial x+\hat{\mathbf{y}} \partial / \partial y+\hat{\mathbf{z}} \partial / \partial z)$ is the gradient, $\nabla_{h}$ is the horizontal component part of $\nabla$, and $\mathbf{u}_{s}$ is the ship maneuvering velocity (in SRF, it is the given forward speed). It should be noted that $\partial \mathbf{u}_{s} / \partial t$ includes both magnitude and direction changes of the maneuvering velocity. In addition, $\mathbf{v}_{h}$ is the horizontal component of translational velocity $\mathbf{v}_{c}$ for surge and sway (absent in the fixed heading scenario).

The impenetrable boundary conditions are

$$
\frac{\partial \varphi}{\partial z}=0
$$


at the flat bottom $z=-H$, and

$$
\hat{\mathbf{n}}_{\Sigma} \cdot \nabla \varphi=\hat{\mathbf{n}}_{\Sigma} \cdot\left[\mathbf{u}_{s}+\mathbf{v}_{c}+\Omega \times\left(\mathbf{x}^{\Sigma}-\mathbf{x}_{c}\right)\right]
$$

on the ship surface $\mathbf{x}^{\Sigma}$. In the above equation, $\hat{\mathbf{n}}_{\Sigma}$ is the normal unit vector of the ship surface $\mathbf{x}^{\Sigma}$, and $\mathbf{x}_{c}$ is the mass center of the ship hull.

2.2. Solid Body Motion Model. The ship solid body motion is decoupled into the translational motion of ship mass center $\mathbf{x}_{c}$ and the rotation about $\mathbf{x}_{c}$. The translational motion, $\mathbf{v}_{c}$, is described by the following equation in MRF:

$$
m_{s} \frac{d \mathbf{v}_{c}}{d t}+D_{\nu} \mathbf{v}_{c}=\mathbf{F},
$$

where $m_{s}$ is the total ship mass and $\mathbf{F}$ is the total force on the ship:

$$
\mathbf{F}=\mathbf{F}^{p}+\mathbf{F}^{g}+\mathbf{F}^{r},
$$

where

$$
\mathbf{F}^{p} \equiv-\iint_{\Sigma_{\text {wet }}} \hat{\mathbf{n}}_{\Sigma} p d \Sigma
$$

is the (driving) pressure force on the ship hull,

$$
\mathbf{F}^{g}=\left(\rho_{w} V_{\text {wet }}-m_{s}\right) \mathbf{g},
$$

is the buoyancy force (restoring force). This force acts only in the vertical direction. But the rudder force $\mathbf{F}^{r}$ in $(4 \mathrm{~b})$ is the new addition. We will discuss this in more detail in the next section. $V_{\text {wet }}$ is under water volume.

The solid body rotation about the ship mass center is governed by the Liouville equation defined in SRF:

$$
\mathbf{I} \cdot \frac{d \boldsymbol{\Omega}}{d t}+\boldsymbol{\Omega} \times(\mathbf{I} \cdot \boldsymbol{\Omega})+D_{\Omega}(\mathbf{I} \cdot \boldsymbol{\Omega})=\mathbf{\Gamma},
$$

where $\mathbf{I}$ is the moment of inertia tensor about the ship mass center $\mathbf{x}_{c}$ (and its components are denoted by $\left.I_{(i, j)}\right), D_{\nu}$ and $D_{\Omega}$ are integrated dissipative effects (e.g., drag) of the fluid on the ship hull, and $\Gamma$ is the total moment (torque) on the ship:

$$
\boldsymbol{\Gamma}=\boldsymbol{\Gamma}^{p}+\boldsymbol{\Gamma}^{g}+\boldsymbol{\Gamma}^{r} .
$$

It includes the (driving) pressure torque:

$$
\Gamma^{p}=-\iint_{\Sigma_{\mathrm{wet}}} d \Sigma\left(\mathbf{x}^{\Sigma}-\mathbf{x}_{c}\right) \times \hat{\mathbf{n}}_{\Sigma} p,
$$

(where $\Sigma_{\text {wet }}$ is total wetted ship surface), the restoring torque:

$$
\Gamma^{g}=\left(\mathbf{x}_{\text {wet }}-\mathbf{x}_{c}\right) \times\left[\left(m_{s}-\rho_{w} V_{\text {wet }}\right) \mathbf{g}\right],
$$

(where $\mathbf{x}_{\text {wet }}$ is the position vector in under water volum), and the torque $\boldsymbol{\Gamma}^{r}$ from the rudder (a new addition). It should be pointed out that the restoring torque is always horizontal, thus affecting only roll and pitch motions.

2.3. Rudder Force and Moment. The rudder force $\mathbf{F}^{r}$ in (4b) and the moment $\Gamma^{r}$ in (5b) are the new physical variables included in this expansion. Since the rudder position is often defined relative to the ship hull, it is therefore very convenient to evaluate them in SRF. Therefore, this leads to the following formulations:

$$
\begin{aligned}
\mathbf{F}^{r} & =\iint_{A} \hat{\mathbf{n}}^{r} p^{r} d s=F_{x}^{r} \widehat{\mathbf{x}}_{s}+F_{y}^{r} \hat{\mathbf{y}}_{s}+F_{z}^{r} \widehat{\mathbf{z}}_{s}, \\
\Gamma^{r} & =\iint_{A} \mathbf{R} \times \hat{\mathbf{n}}^{r} p^{r} d s+\mathbf{R}^{t} \times \mathbf{F}^{\Omega} \\
& =\Gamma_{x}^{r} \widehat{\mathbf{x}}_{s}+\Gamma_{y}^{r} \hat{\mathbf{y}}_{s}+\Gamma_{z}^{r} \widehat{\mathbf{z}}_{s}+\left(\mathbf{x}-\mathbf{x}_{C}\right) \times \mathbf{F}^{\Omega},
\end{aligned}
$$

where $p^{r}$ is the potential flow pressure; but $A=A^{\Sigma}-A^{\text {block }}$ ( $A^{\Sigma}$ is the wetted rudder surface, and $A^{\text {block }}$ is the blocking effected area) [16], $\hat{\mathbf{n}}^{r}$ is the unit normal vector of $A, \mathbf{x}_{C}$ is the position vector of the center of the circle tangent to the point $\mathbf{x}$ on the ship trajectory, $\mathbf{R}^{t}$ is the radius vector of ship trajectory to the point $\mathbf{x}$, and

$$
\mathbf{R} \equiv R_{x} \widehat{\mathbf{x}}_{s}+R_{y} \widehat{\mathbf{y}}_{s}+R_{z} \widehat{\mathbf{z}}_{s}
$$

is the rotating arm vector of the rudder. The force $\mathbf{F}^{\Omega}$ in (7) describes the effect of maneuvering directional changes in SRF and is, therefore, dependant on the changes in the rudder angle $\boldsymbol{\delta}$ (defined relative to the ship hull):

$$
\begin{gathered}
\mathbf{F}^{\Omega}=\iint_{A} \rho\left[2 \boldsymbol{\Omega}^{*} \times \mathbf{v}_{c}+\boldsymbol{\Omega}^{*} \times\left(\boldsymbol{\Omega}^{*} \times \mathbf{x}\right)+\frac{d \boldsymbol{\Omega}^{*}}{d t} \times \mathbf{x}\right] d s, \\
\boldsymbol{\Omega}^{*}=\frac{d \boldsymbol{\beta}}{d t}+\Omega_{z} \hat{\mathbf{z}}_{s}
\end{gathered}
$$

where $\beta$ is the drifting angle vector (the angle between the ship's horizontal velocity vector and the ship's longitudinal axis). If the rudder can be approximated as a plate rotating about the vertical direction of the ship hull with a rudder deflection angle $\delta$, the horizontal components of $\mathbf{F}^{r}$ can be substantially simplified:

$$
\begin{aligned}
& F_{x}^{r}=-C_{l} \frac{1}{2} \rho u_{r}^{2} A \sin ^{2} \delta+\iint_{A} \hat{n}_{x}^{r} p_{w}^{r} d s, \\
& F_{y}^{r}=C_{l} \frac{1}{4} \rho u_{r}^{2} A \sin 2 \delta+\iint_{A} \hat{n}_{y}^{r} p_{w}^{r} d s,
\end{aligned}
$$

where $u_{r}$ is the effective inflow speed; $\hat{n}_{x}^{r}$ and $\hat{n}_{y}^{r}$ are the $x$ and $y$ components of the normal vector of the rudder surface, respectively; $p_{w}^{r}$ is pressure on rudder surface due to the incident waves; $C_{l}$ is the lift curve slope:

$$
C_{l}=\frac{2 \pi}{1+(2 /(L / d))},
$$

where $L$ and $d$ are the length and the width of the rudder, respectively [21]. This theory has been tested by many experiments and is broadly used in ship dynamics. It should be pointed out that the separation effects are included by using $A$ (not $A^{\Sigma}$ ) in (6)-(10).

By (10), the vertical moment is

$$
\begin{aligned}
\Gamma_{z}^{r} & =R_{x} F_{y}^{r}-R_{y} F_{x}^{r} \approx R_{x} F_{y}^{r} \\
& =\left(C_{1} \frac{1}{4} \rho u_{r}^{2} A \sin 2 \delta+\iint_{A} \hat{n}_{y}^{r} p_{w}^{r} d s\right)\left(x_{r}-x_{c}\right),
\end{aligned}
$$


since $R_{y}$ is in general much smaller than $R_{x}$. In (12), $\left(x_{r}-x_{c}\right)$ is the longitudinal distance between the ship mass center $x_{c}$ and the rudder mass center $x_{r}$.

The effective inflow into the rudder is derived from the ship forward speed and the accelerated flow in the propeller slipstream. The latter depends on the propeller's mechanical design. In the cases discussed in this paper, we choose an empirical estimate

$$
\mathbf{u}_{r}=1.6 \mathbf{u}_{s},
$$

from experimental data for naval vessels with Froude numbers ranging from 0.2 to 0.4 [22].

2.4. Ship Track Kinematics. Kinematics of ship track variation can be best described in an inertial reference frame, for example, the earth-fixed reference frame (ERF) $\left(\hat{\mathbf{x}}_{e}, \hat{\mathbf{y}}_{e}, \hat{\mathbf{z}}_{e}\right)$. The ship moving direction angle, $\theta^{*}$, is the sum of the yaw angle $\theta_{z}$ (the rotational angle in $z$ direction, and $d \theta_{z} / d t=\Omega_{z}$ obtained from (5a)-(5d)) and the drift angle $\beta$ :

$$
\begin{aligned}
\theta^{*} & =\theta_{z}+\beta, \\
\beta & \equiv \tan ^{-1}\left(\frac{\nu_{y}}{\left|\mathbf{u}_{s}\right|+\nu_{x}}\right),
\end{aligned}
$$

where $v_{x}$ and $v_{y}$ are ship translation motion in the $x$ and $y$ directions in ship coordinate, respectively. The sketch map can be find in Figure 5 page 198 [23, Figure 5, page 198]. The track $\mathbf{x}_{\text {track }}$ of the ship in ERF is defined as

$$
\mathbf{x}_{\text {track }}=x_{\text {track }} \hat{\mathbf{x}}_{e}+y_{\text {track }} \hat{\mathbf{y}}_{e} .
$$

In particular,

$$
\frac{d \mathbf{x}_{\text {track }}}{d t}=\mathbf{A}_{m} \cdot\left(\mathbf{u}_{s}+\mathbf{v}_{h}\right) \equiv \mathbf{u}, \quad \mathbf{A}_{m}=\left[\begin{array}{cc}
\cos \theta_{z} & -\sin \theta_{\mathrm{z}} \\
\sin \theta_{z} & \cos \theta_{\mathrm{z}}
\end{array}\right]
$$

If the rudder deflection angle $\delta$ is given, then the rudder force (10) and moment (12) can be determined. If the ship velocity is calculated, for example, via (4a)-(4d) and (5a)(5d), then the track can be determined via time integration of (16). On the other hand, given the navigation history, that is, the ship track $\mathbf{x}_{\text {track }}(t)$, the total ship horizontal velocity, $\mathbf{u}$, and horizontal acceleration can be calculated by simple time differentiation, and the direction angle $\theta^{*}$ can be also determined via

$$
\theta^{*}=\tan ^{-1}\left(\frac{\mathbf{u} \cdot \hat{\mathbf{y}}_{e}}{\mathbf{u} \cdot \hat{\mathbf{x}}_{e}}\right)
$$

The the rudder angle $\delta$ can be obtained from horizontal component of $(4 a)-(4 d)$, vertical component of $(5 a)-(5 d)$, (10), and (16) using an iterative method. Finally we want to point out that, in DiSSEL ship motion model, hydrodynamic interactions between the hull and the rudder are properly implemented, in addition to the implementation of the interactions of the six-degree freedom of the ship motions. These nonlinear interactions are obtained by solving a set of the nonlinear equations (1)-(7).

2.5. Centrifugal Effect. Ship track direction changes generate a finite centrifugal force. In a very simple mathematical description, the force can be described as a vertical rotation about the arc center $C$ of the track curve:

$$
\mathbf{u}_{s}=\omega \hat{\mathbf{z}}_{e} \times\left(\mathbf{x}_{\text {track }}-\mathbf{x}_{C}\right),
$$

where $\omega$ is the rotation speed about $C$ and $\mathbf{x}_{C}$ is the position vector of the point $C$ in ERF. Therefore,

$$
\frac{\partial \mathbf{u}_{s}}{\partial t}=\omega^{2} \widehat{\mathbf{z}}_{e} \times\left[\widehat{\mathbf{z}}_{e} \times\left(\mathbf{x}_{\text {track }}-\mathbf{x}_{C}\right)\right] .
$$

Thus,

$$
\frac{p}{\rho}+\frac{\partial \mathbf{u}_{s}}{\partial t} \cdot \mathbf{x}_{\text {track }}=\frac{1}{\rho}\left[p+\frac{1}{2} \rho \omega^{2}\left|\hat{\mathbf{z}}_{e} \times\left(\mathbf{x}_{\text {track }}-\mathbf{x}_{C}\right)\right|^{2}\right] \equiv \frac{\tilde{p}}{\rho} .
$$

In other words, the changes in the direction of the ship motion can be described by the effective pressure $\tilde{p}$ in (20). Given the ship track $\mathbf{x}_{\text {track }}(t)$, the radius $\left|\mathbf{x}_{\text {track }}-\mathbf{x}_{C}\right|$ can then be calculated with the standard curvature formulation:

$$
\left|\mathbf{x}_{\text {track }}-\mathbf{x}_{C}\right|=\frac{1}{\kappa(t)}, \quad \kappa(t)=\frac{\left|\left(d x_{\text {track }} / d t\right)\left(d^{2} y_{\text {track }} / d t^{2}\right)-\left(d y_{\text {track }} / d t\right)\left(d^{2} x_{\text {track }} / d t^{2}\right)\right|}{\left[\left(d x_{\text {track }} / d t\right)^{2}+\left(d y_{\text {track }} / d t\right)^{2}\right]^{3 / 2}}
$$

Its influence on the solid body rotation of the ship can be computed via integration of the effective pressure over the ship surface.

\section{Stable and Conservative Algorithm for Ship Maneuvering}

When a ship maneuvers in seaway, its direction and speed change over time. This leads to time-varying encounter waves, thus potentially to numerical difficulties in conserving the momentum of the entire system. This will be a source of numerical instabilities. Therefore, we will discuss first the nature of the numerical difficulties (based on current ship motion models) and then introduce our new stable algorithm that conserves the momentum of the system.

3.1. Dynamical Imbalance from Rapidly Changing Encounter Frequencies. As pointed out by Lin and Kuang [24], the numerical balance between the driving forces and the restoring forces is the key to model correctly ship motions. 
Lin and Kuang [1] further showed that DiSSEL can well maintain such dynamic balances for fixed heading of various ship hulls in a wide range of environments.

To expand DiSSEL for modeling ship maneuvering, a natural consideration is whether the algorithm used in the model is able to maintain accurately the dynamic balance between the driving forces and the restoring forces on the ship hull when ship heading changes in time. The consideration arises from the very fact that such changes also alter the environment observed on the ship. For example, in SRF, a surface wave can be generally described as

$$
\eta(x, y, t)=\sum_{i=1}^{N} \sum_{j=1}^{M} A_{i j} \sin \left\{\mathbf{k}_{i j} \cdot\left(\mathbf{x}-\mathbf{x}_{c}\right)+\omega_{i j}^{\mathrm{en}} t+\phi_{i j}\right\}
$$

where $A_{i j}$ is the amplitude of the mode $(i, j), \mathbf{k}_{i j}$ is the wave number vector, $\omega_{i j}^{\mathrm{en}}$ is the encounter frequency, and $\phi_{i j}$ is the phase. The integer pair $(M, N)$ defines the total number of the wave modes used in numerical simulation. The encounter frequency $\omega_{i j}^{\mathrm{en}}$ is defined as

$$
\omega_{i j}^{\mathrm{en}}=\omega_{i j}^{\mathrm{sea}}\left[1-\left|\frac{\mathbf{u}}{\mathbf{c}_{i j}}\right| \cos \alpha_{i j}^{\mathrm{sea}}\right] \text {, }
$$

where $\mathbf{c}_{i j}$ is the phase velocity of the wave mode, $\alpha_{i j}^{\text {sea }}$ is the angle between $\mathbf{c}_{i j}$ and $\mathbf{u}$, and $\omega_{i j}^{\text {sea }}$ is the incident wave frequency in ERF. For any given incident wave, $\alpha_{i j}^{\text {sea }}$ changes with the ship heading direction.

One could clearly observe the complications and its numerical difficulties from (23): changes in $\mathbf{v}_{h}$ and $\theta_{z}$ (and therefore $\alpha_{i j}^{\text {sea }}$ ) are dynamically determined via the driving forces and the restoring forces on the ship hull which depend on the encounter frequency $\omega_{i j}^{\text {en }}$. But changes in $\omega_{i j}^{\text {en }}$ depend also on $\mathbf{v}_{h}$ and $\theta_{z}$. Therefore, errors in the force balances could potentially lead to numerical instabilities and false numerical solutions in ship motion simulation.

To help understand this, we consider a very mathematically simple case: a ship maneuvering in a single environment wave $(M=N=1)$ (and is called Case 1 in the rest of the paper). The ship heading direction changes at a steady rate (with the rudder angle $\delta=15^{\circ}$ ). The value of the ship speed is also fixed and slow, with the Froude number $F_{r}=0.266$. We first model this simple system with the existing version of DiSSEL [1]. The numerical driving forces (moments) and the restoring forces (moments) for heave, pitch, and roll are shown in Figure 1. From the figure one can observe that, as soon as the ship direction starts to change, the balance between the forces (moments) is lost numerically.

What is the cause of this imbalance? One possibility is the time variation of the encounter frequency $\omega^{\mathrm{en}}$. To verify this, we consider Case 2: an artificial system in which $\omega^{\text {en }}$ is fixed, but the ship heading direction change remains the same as in the previous case. Numerical results for this case are shown in Figure 2. Clearly, the force balance is well maintained for this artificial system. Of course, this is not a mathematical proof, but it is sufficient to demonstrate the key problem for retaining numerical force balances in ship motion modeling.

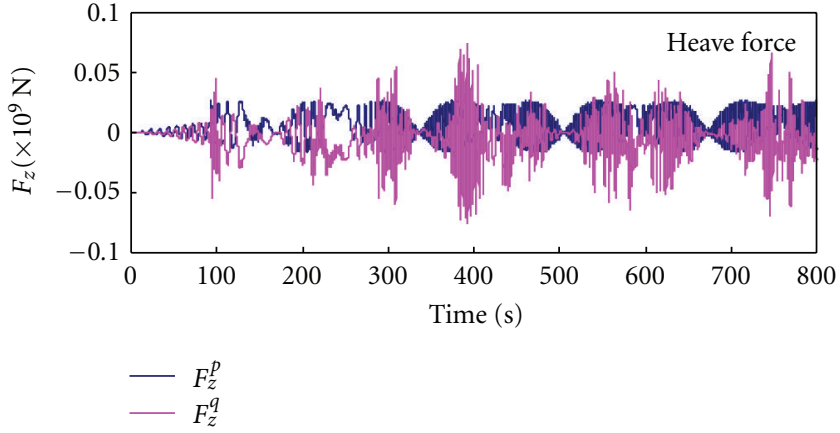

(a)

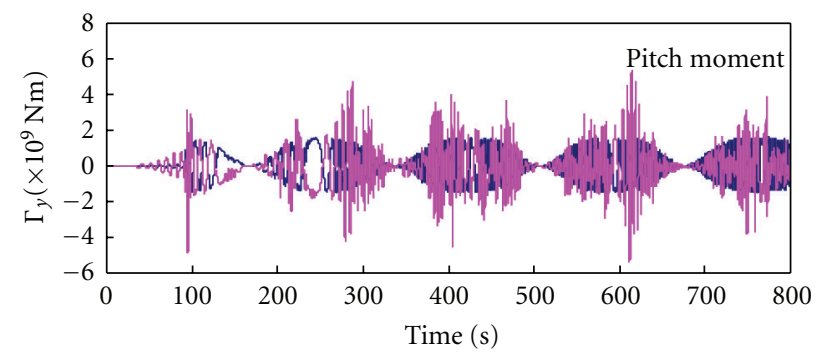

$-\Gamma_{y}^{p}$

(b)

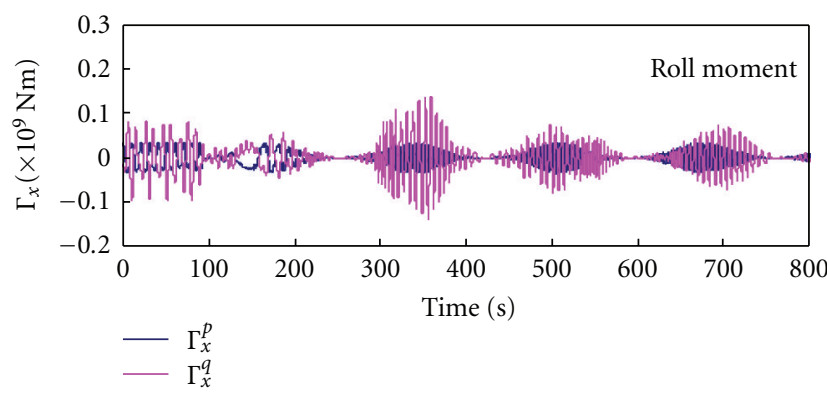

(c)

FIgURE 1: The numerical driving and restoring forces (moments) for heave (a), pitch (b), and roll (c) from the original DiSSEL. The ship hull is EHF, with a rudder angle $\delta=15^{\circ}$ and the Froude number $F_{r}=0.266$. The incident wave height is $w h=1.496 \mathrm{~m}$, and its wavelength normalized by ship length is $\lambda=1.0$ (Case 1). The superscripts " $p$ " and " $q$ " represent the driving forces (moments) and restore forces (moments) which associated with (4c), (4d), and $(5 c)$, and (5d).

3.2. Adaptive Method for the Time-Varying Encounter Frequencies. The above numerical experiments can guide our effort in developing a new algorithm to maintain dynamic balance for time-varying encounter frequencies. In particular, we intend to exploit the fact that the force balances can be well achieved for a steady encounter frequency.

A simple and effective approach is to apply the same numerical algorithm in a "fictitious" reference frame in which the encounter frequency is time invariant. To explain this approach, we will start from a single-wave scenario. 


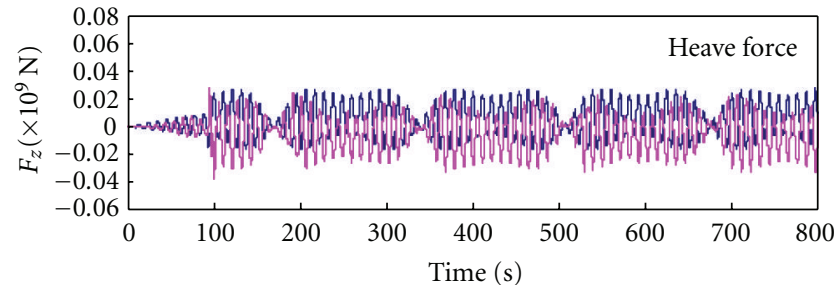

$-F_{z}^{p}$

(a)

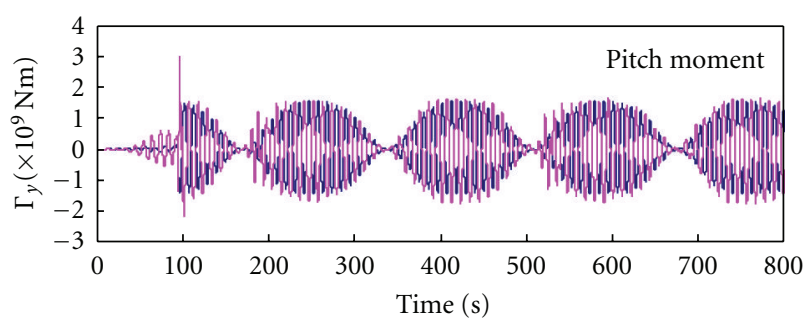

$-\Gamma_{y}^{p}$

(b)

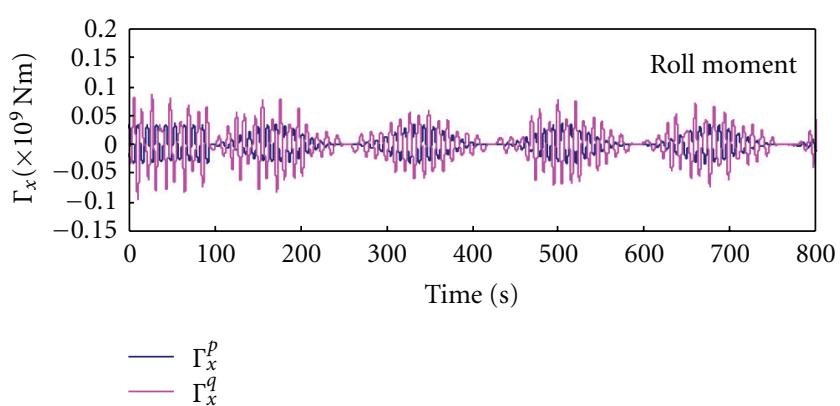

(c)

FIgure 2: Same as Figure 1, but with the encounter frequency fixed to $\omega^{\text {en }}=\omega^{\text {sea }}($ Case 2$)$.

In this case, we introduce a "fictitious" time $t^{*}$ and its corresponding time step $\Delta t^{*}$, such that

$$
\left|\frac{\omega^{\mathrm{en}}(t) \Delta t}{\mathbf{k}}\right|=\left|\frac{\omega^{\mathrm{sea}} \Delta t^{*}}{\mathbf{k}}\right| \text {. }
$$

From (23),

$$
\Delta t^{*}=|1-| \frac{\mathbf{u}}{\mathbf{c}}\left|\cos \alpha^{\mathrm{sea}}\right| \Delta t
$$

But (25) could lead to $\Delta t^{*}=0$ when

$$
\left[1-\left|\frac{\mathbf{u}}{\mathbf{c}}\right| \cos \alpha^{\text {sea }}\right]=0,
$$

that is,

$$
|\mathbf{c}|=|| \frac{\mathbf{u}}{\mathbf{c}}\left|\cos \alpha^{\mathrm{sea}}\right|
$$
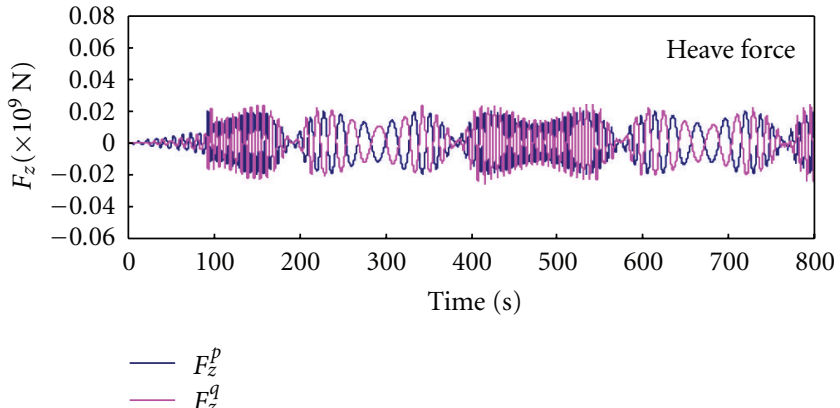

(a)

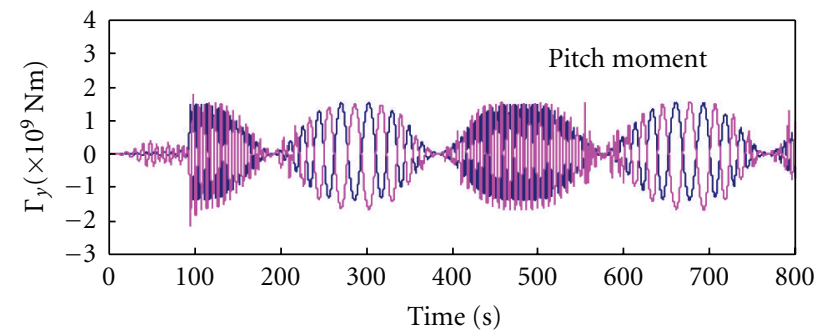

$-\Gamma_{y}^{p}$

(b)

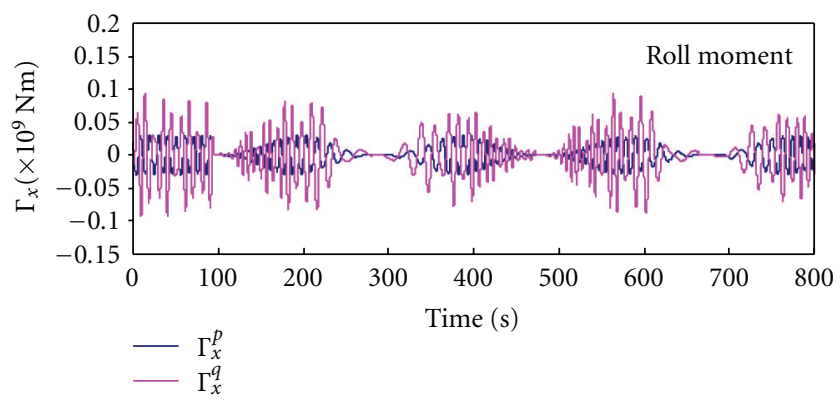

(c)

FIgure 3: Same as Figure 1 (Case 1), but the forces are calculated by the new algorithm defined in (28) and (29a)-(29b).

To avoid this numerical singularity, we modify (25) by adding a truncation limit:

$$
\Delta t^{*}=\left\{\begin{array}{l}
\Delta \tau^{*} \equiv \varepsilon, \quad \text { if }|1-| \frac{\mathbf{u}}{\mathbf{c}}\left|\cos \alpha^{\text {sea }}\right| \Delta t \leq \varepsilon \\
1-\left|\frac{\mathbf{u}}{\mathbf{c}}\right| \cos \alpha^{\text {sea }} \mid \Delta t, \Delta \tau^{*}=0, \text { otherwise }
\end{array}\right.
$$

Substituting (28) into (22) and setting $M=N=1$ (singlemodel wave), we have

$$
\eta(x, y, t)=A \sin \left\{\mathbf{k} \cdot\left(\mathbf{x}-\mathbf{x}_{c}\right)+\mu \omega^{\mathrm{sea}}\left(t^{*}-\tau^{*}\right)\right\},
$$

where

$$
\mu=\operatorname{sgn}\left\{1-\left|\frac{\mathbf{u}}{\mathbf{c}}\right| \cos \alpha^{\mathrm{sea}}\right\}
$$

and $\tau^{*}=\sum \Delta \tau^{*}, t^{*}=\sum \Delta t^{*}$. 

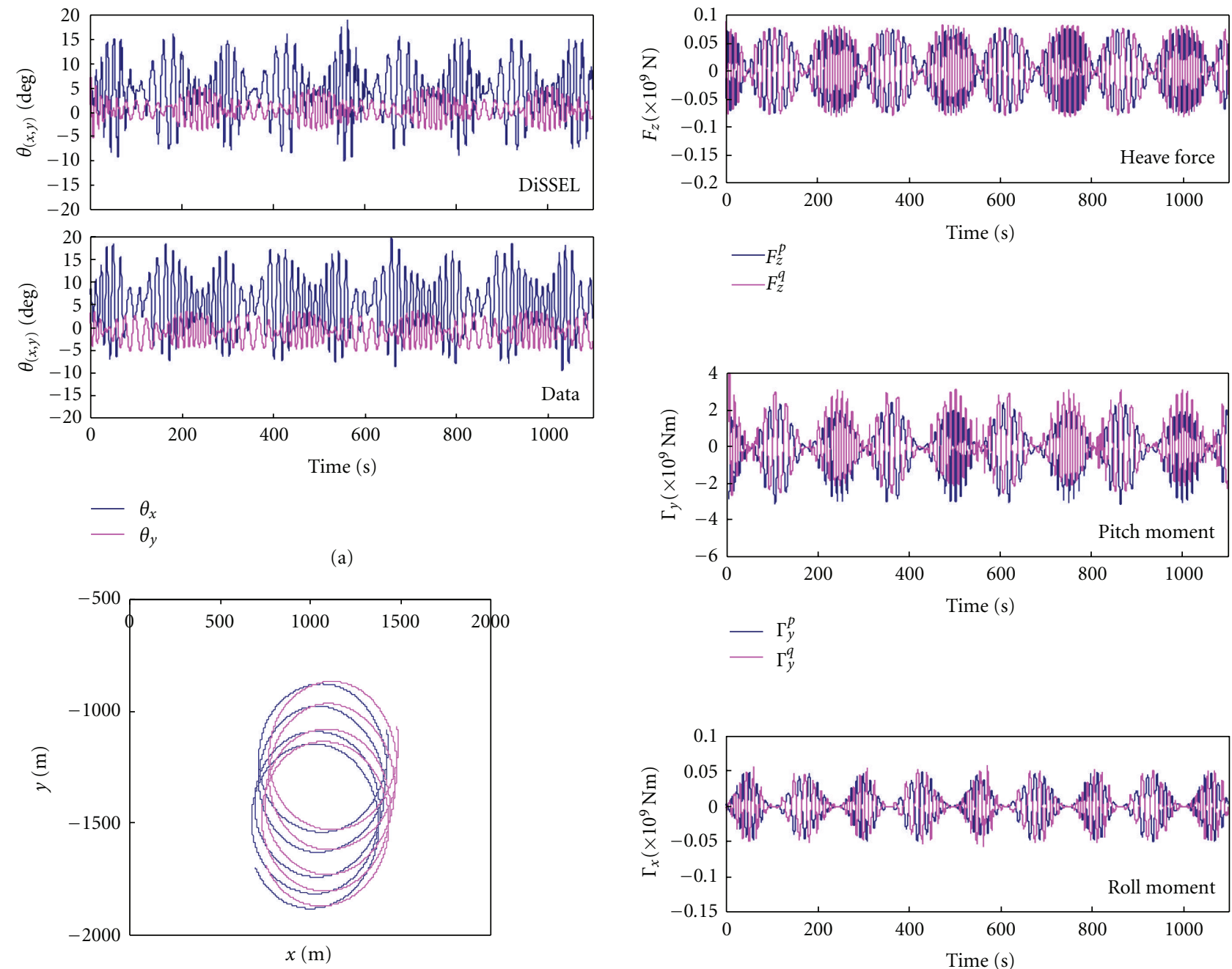

(a)
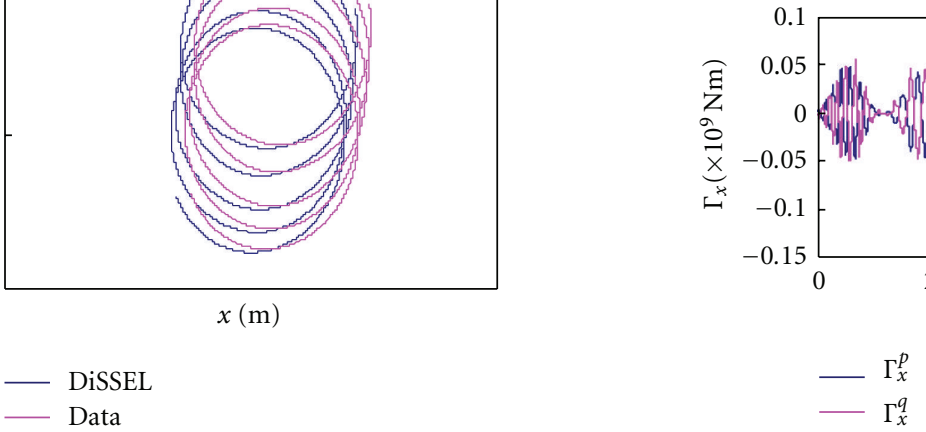

(b)

(c)

FIGURE 4: (a) Roll and pitch motions of EHF with $\delta=30^{\circ}, F_{r}=0.2146$ in the regular incident wave $w h=5.984 \mathrm{~m}, \lambda=2.0$ (Case 3 ). The numerical results from the improved DiSSEL are on the top, and the experimental data are in the bottom. (b) Same as (a), but for the ship track in Earth coordinate, $x$ is the initial direction, and $y$ is perpendicular to $x$ direction. (c) Same as (a), but for the driving and restoring forces (moments).

To test the applicability of this approach, we reexamine Case 1. The force balance is now well retained with the new algorithm as shown in Figure 3. By comparing this with Figure 1, we can observe clearly the improvement by the new algorithm.

We test further the new algorithm with more complicated cases that have experimental data. Due to space limitations, we show here only two of them: the EHF maneuvering in regular waves with the largest rudder angle $\delta=30^{\circ}$ (Case 3) and with the fastest forward speed $F_{r}=0.368$ (Case 4). The results for the two cases are shown in Figures 4 and 5. Because in the experiments, the ship motions, wave elevations, and trajectory motions were collected by separate systems, all the data sets could not be aligned in time accurately enough to know the initial conditions of the system. Therefore, the benchmarking comparison focuses only on the overall characteristics of the motion and not a point-by-point prediction comparison. As shown in these figures, our numerical results agree very well with the experimental data. The good agreement is because the dynamical balances are now correctly modeled in the simulation, as shown in Figures 4(c) and 5(c).

This new algorithm can be easily extended to arbitrary two-dimensional irregular waves. The mathematical details are shown as follows: expression (29a) is now modified to irregular waves:

$$
\begin{array}{r}
\eta(x, y, t)=\sum_{i=1}^{N} \sum_{j=1}^{M} A_{i j} \sin \left\{\mathbf{k}_{i j} \cdot\left(\mathbf{x}-\mathbf{x}_{c}\right)+\omega_{i j}^{\mathrm{en}} t+\phi_{i j}\right\} \\
=\sum_{i=1}^{N} \sum_{j=1}^{M} A_{i j} \sin \left\{\mathbf{k}_{i j} \cdot\left(\mathbf{x}-\mathbf{x}_{c}\right)+\phi_{i j}\right. \\
\left.+\mu \omega_{i j}^{\text {sea }}\left(t_{i j}^{*}-\tau_{i j}^{*}\right)\right\},
\end{array}
$$



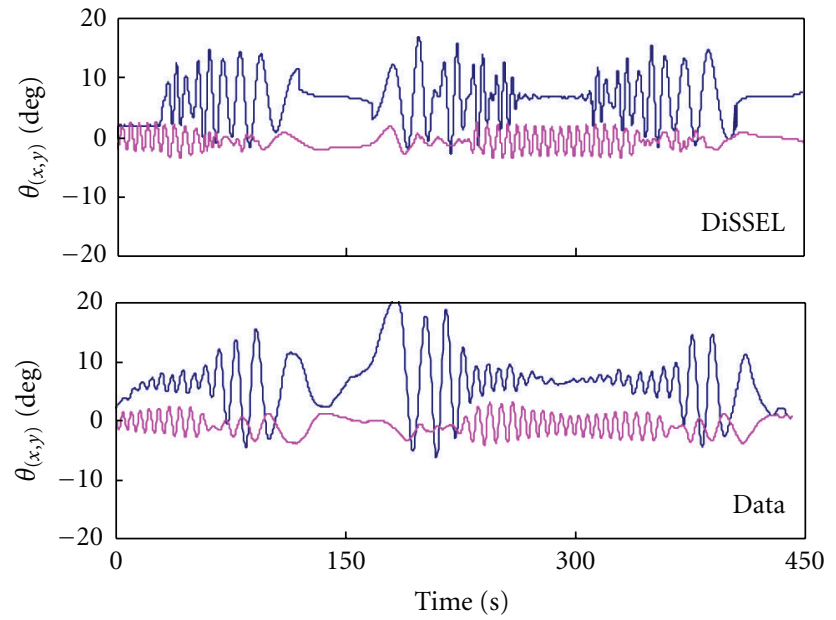

$\theta_{x}$
$-\theta_{y}$

(a)

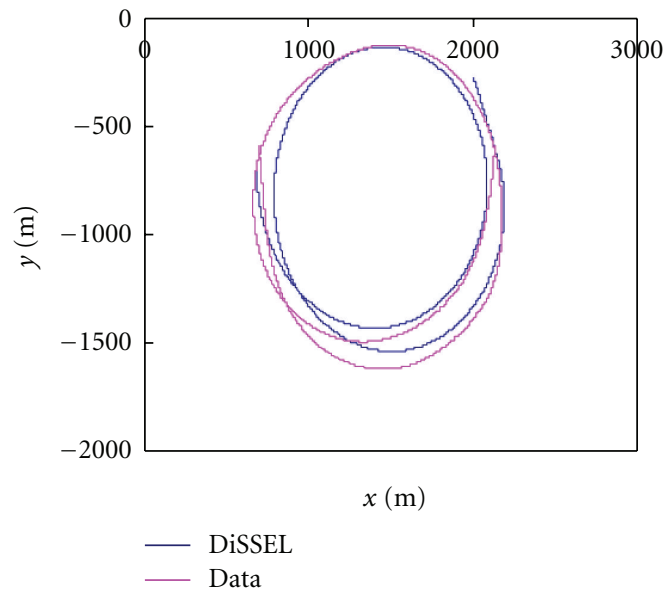

(b)
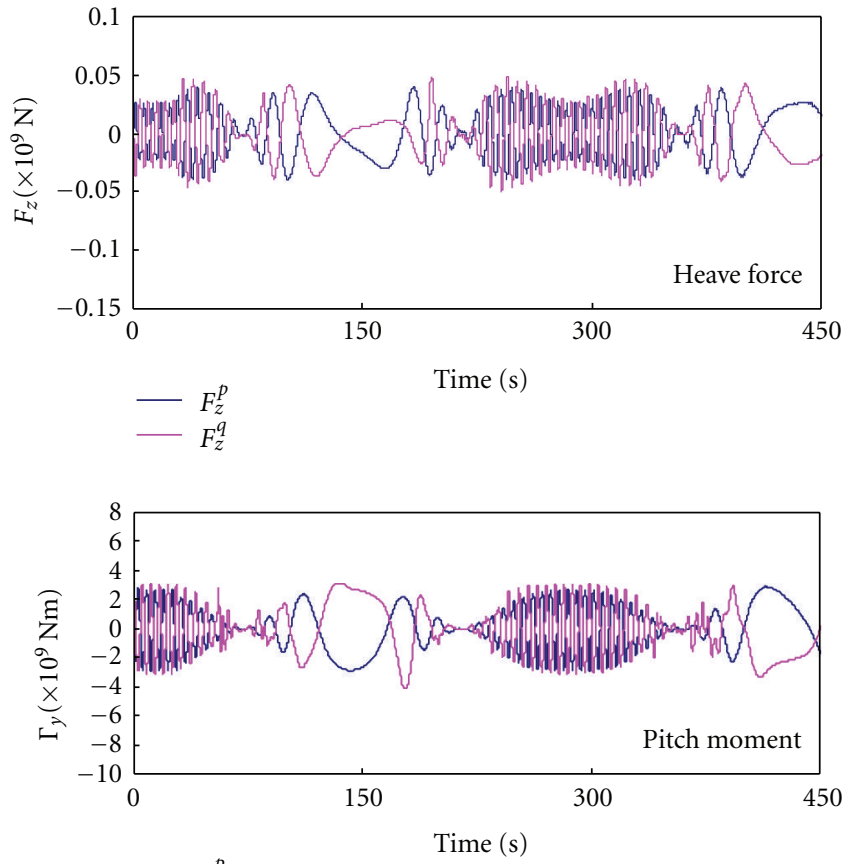

$-\Gamma_{y}^{p}$

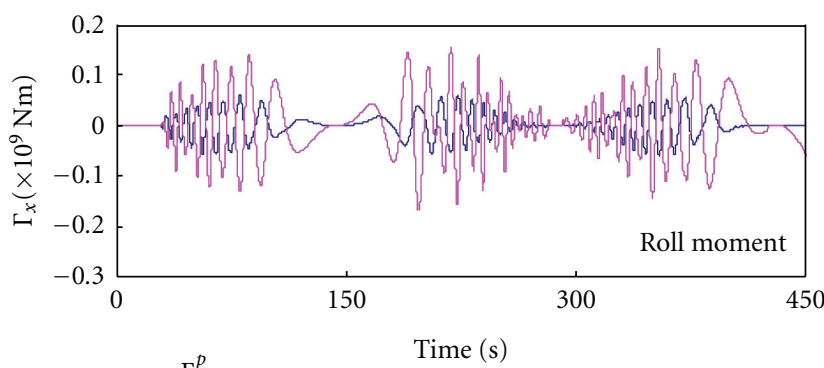

$-\Gamma_{x}^{p}$

Time (s)

(c)

Figure 5: (a) Same as Figure 4(a), but for the case $F_{r}=0.368, \delta=14.4^{\circ}, w h=2.992 \mathrm{~m}, \lambda=1.0$ (Case 4). (b) Same as Figure 4(b), but for Case 4. (c) Same as Figure 4(c), but for Case 4.

where $\Delta t_{i j}^{*}$ and $\Delta \tau_{i j}^{*}$ are defined similarly as in (28) for each wave mode $\mathbf{c}_{i j}$ and $\alpha_{i j}^{\text {sea }}$.

To maintain computational efficiency, we introduce an effective incident wave frequency and wave amplitude:

$$
\begin{gathered}
\omega^{\text {sea }} \equiv \frac{\sum_{i j}^{M, N} A_{i j} \omega_{i j}^{\text {sea }}}{A^{\Sigma}}, \\
A^{\Sigma} \equiv \sum_{i j}^{M, N} A_{i j} .
\end{gathered}
$$

With these definitions, we can then define a single effective "fictitious" time step

$$
\Delta t^{*} \equiv \frac{1}{\omega^{\text {sea }} A^{\Sigma}} \sum_{i, j}^{M, N} A_{i j} \omega_{i j}^{\text {sea }} \Delta t_{i j}^{*} .
$$

The notions of the effective incident wave frequency and the "fictitious" time are the same as those in the one-dimensional regular wave formulations. But the actual physical meanings are different. In particular, $\omega^{\text {sea }}$ is given and invariant throughout the simulation. But $\Delta t^{*}$ changes in time because of time-varying $\Delta t_{i j}^{*}$. Therefore, (30) can be rewritten as

$$
\begin{aligned}
& \eta(x, y, t) \\
& \quad=\sum_{i=1}^{N} \sum_{j=1}^{M} A_{i j} \sin \left\{\mathbf{k}_{i j} \cdot\left(\mathbf{x}-\mathbf{x}_{c}\right)+\phi_{i j}+\mu \omega_{i j}^{\text {sea }}\left(c_{i j}^{*} t^{*}-\tau_{i j}^{*}\right)\right\},
\end{aligned}
$$

where

$$
c_{i j}^{*} \equiv \frac{t_{i j}^{*}}{t^{*}}
$$




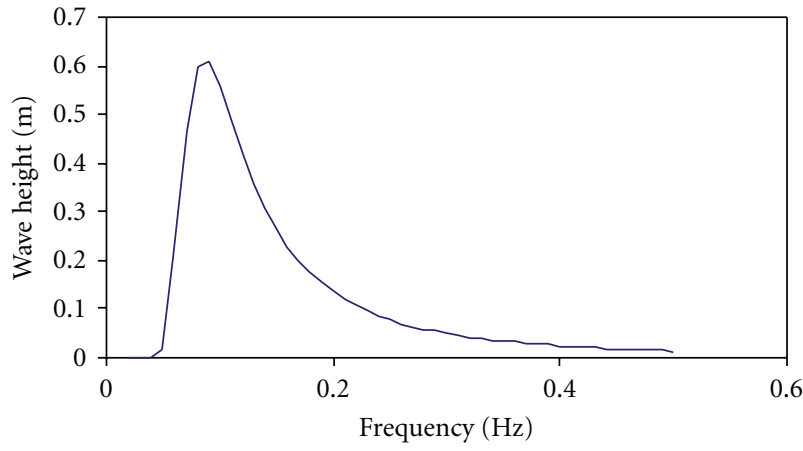

FIGURE 6: Irregular incident wave spectrum with the peak frequency $\omega_{p}=0.586$ and the significant wave height, $\mathrm{SWH}=6 \mathrm{~m}$.

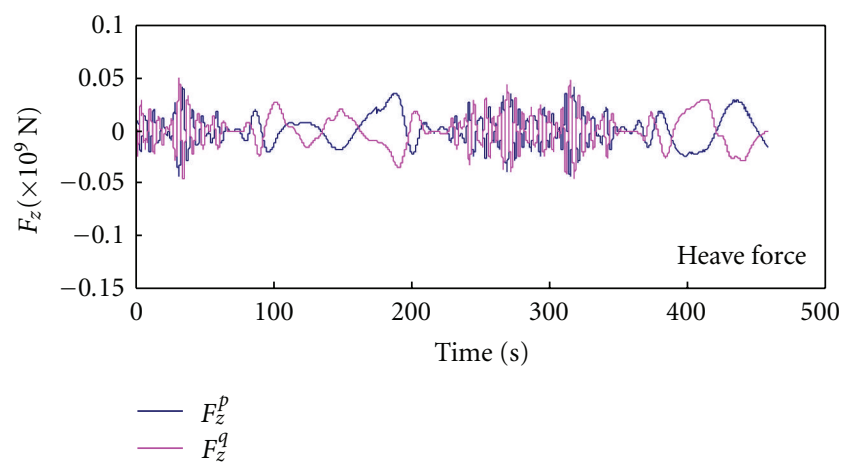

(a)

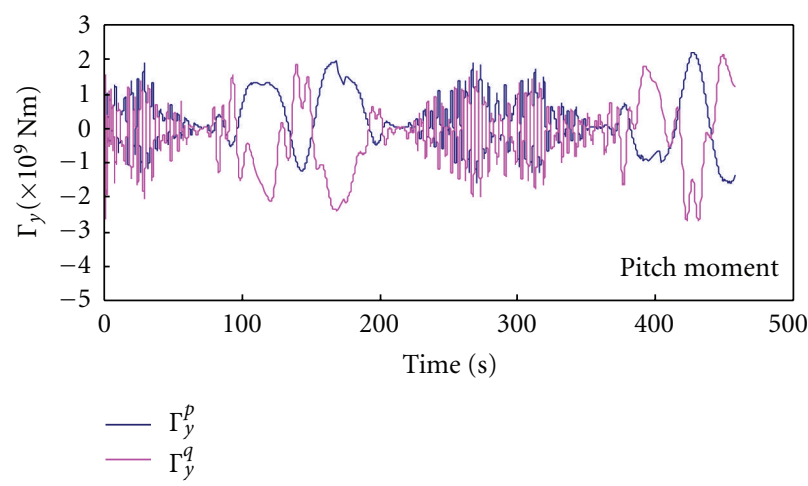

(b)

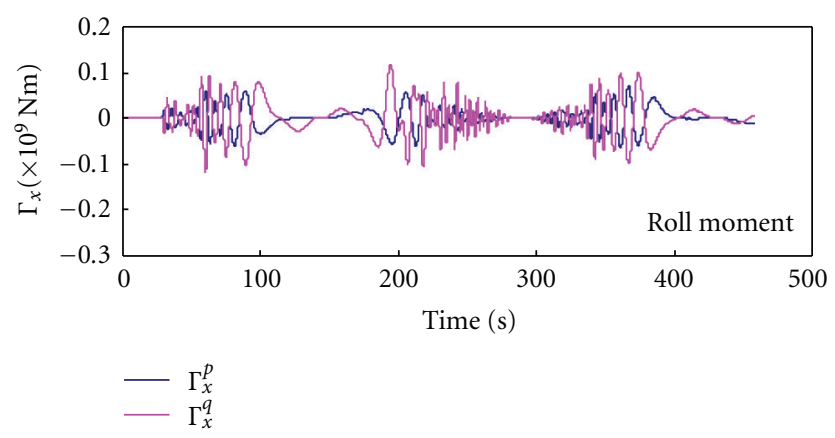

(c)

Figure 7: The numerical driving and restoring forces (moments) from the improved DiSSEL for Case 4, but with the irregular incident waves specified in Figure 6.
Again, $\Delta t$ and $\Delta t_{i j}^{*}$ (and therefore $\Delta t^{*}$ by definition) must satisfy the CFL condition in the simulation.

It should be pointed that this method is more efficient than iterative methods not only because it calculates the mean $\Delta t_{i j}^{*}$ once in each time step, but also because it does not impose any convergence requirement and thus ensures a stable solution.

Since there is no experimental data on ship maneuvering in irregular seas with which to compare, we will only examine the dynamical balances in the numerical solutions, with no attempt made for benchmarking purposes. For this, we reconsider Case 4, but replace the regular wave with the irregular wave specified by the spectral shape in Figure 6 . The numerical results are summarized in Figure 7. From the figures we can observe that the dynamical balances are well established in the numerical solutions for roll, pitch, and heave motions, that is, the driving forces (moments) are comparable in magnitude and opposite in sign to the restoring forces (moments).

\section{Conclusion}

When a ship maneuvers in a seaway, arbitrary incident waves measured on the ship are time varying (i.e., time-varying encounter frequencies). This time variation depends on the ship motion state, which, in turn, changes in time under the influence of the incident waves. Therefore, it is critical to maintain the dynamical balances between the driving forces (moments) and the restoring forces (moments) in a numerical simulation for the accurate modeling of ship motion. Such dynamical balance depends on the appropriate evaluation of the incident waves and the corresponding forces on the ship hull and thus requires well-designed numerical approaches to model the nonlinear dependencies between the environment and the ship motion.

We have demonstrated numerically that direct time integration of the ship-wave interactions cannot retain properly the balance when the ship heading changes in time. This heading change results in time-varying encounter wave frequencies. We anticipated that this time-varying encounter frequency could lead to phase misfits between the driving forces and the restoring forces, thus destroying the dynamical balances in the simulation and therefore the numerical stability. This is supported by our numerical simulations of Cases 1 and 2 (shown in Figures 1 and 2).

To eliminate the time variation of the encounter frequency (mainly due to ship heading direction change), we developed a new adaptive algorithm with a mathematical (but "fictitious") time $t^{*}$ defined in (32). This is equivalent to rescaling the numerical time (and thus improving numerical temporal resolution) dynamically with the ship motion states. In this new system, the encounter frequencies are steady as measured in $t^{*}$. We have tested this new algorithm for many cases. All numerical results show that the dynamical balances between the driving forces (moments) and the restoring forces (moments) are well established.

This new algorithm has been implemented in the DiSSEL nonlinear ship motion model. With this improved model, we are able to reproduce satisfactorily the results from all known 
laboratory experiments. Results for two of such experiments are shown in Figure 4 (with the largest rudder angle) and Figure 5 (with the largest forward speed) in regular waves. We also tested the improved model for ship maneuvering in irregular waves specified in Figure 6, and the dynamical balances are retained as well, as shown in Figure 7.

\section{Acknowledgments}

The authors thank Terry Applebee, Y. T. Shen, and J. Gorski for their comments and suggestions to this study. They also thank the Seakeeping Division at NSWCCD for providing the experimental data to benchmark and validate their model. This work is supported by Naval Surface Warfare Center Independent Laboratory In-House Research (ILIR) Program.

\section{References}

[1] R.-Q. Lin and W. Kuang, "Solid-body motion in fully nonlinear shipmotion model," Proceedings of the Royal Society A. In press.

[2] H. R. Chaplin, "Wind-tunnel tests of low-aspect-ratio control surfaces with trailing edge to wind," Tech. Rep. 944, David Taylor Model Basin Report, 1954.

[3] L. W. Folger, D. E. Whicker, and L. F. Fehlner, "Free-stream characteristics of a family of low-aspect-ratio, all-movable control surfaces for application of ship design," Tech. Rep. 933, David Taylor Model Basin Report, 1958.

[4] H. Söding, "Prediction of ship steering capabilities," Schiffstechnik, vol. 29, no. 1, pp. 3-29, 1982.

[5] J. Hooft and J. B. Pieffers, "Maneuverability of frigates in waves," Marine Technology, vol. 25, no. 4, pp. 262-271, 1988.

[6] J.-T. Lee, "A potential based panel method for the analysis of marine propellers in steady flow," Tech. Rep. 87-13, Department of Ocean Engineering, MIT, 1988.

[7] P. A. Bailey, W. G. Price, and P. Temarel, "A Unified mathematical model descriping the manuoeuvring of a ship travelling in a seaway," The Royal Institution of Naval Architects -RINA, pp. 131-149, 1997.

[8] S.-W. Chau, "Computation of rudder force and moment in uniform flow," Ship Technology Research, vol. 45, no. 1, pp. 313, 1998.

[9] O. M. El Moctar, "Numerical determination of rudder forces," in Proceedings of the European Mechanics Society (Euromech '98), vol. 374, Poitiers, France, 1998.

[10] H. Söding, "Limits of potential theory in rudder flow predictions," in Proceedings of the 22nd Symposium on Naval Hydrodynamics, Weinblum Lecture, pp. 622-637, Washington, DC, USA, 1999.

[11] R. Kimoto, T. Katayama, and Y. Ikeda, "Effects of running attitude on hydrodynamic forces for oblique towed planing craft," in Proceedings of the 2nd Asia-Pacific Workshop on Hydrodynamics (APHydro '04), pp. 115-122, Busan, Korea, 2004.

[12] T. Katayama, R. Kimoto, and Y. Ikeda, "Effects of running attitudes on maneuvering hydrodynamic forces for planing hull," in Proceedings of the International Conference on Fast Sea Transportation (FAST '05), St. Petersburg, Russia, 2005.

[13] T. Katayama, T. Taniguchi, H. Fujii, and Y. Ikeda, "Development of maneuvering simulation method for high speed craft using hydrodynamic forces obtained from model tests," in Proceedings of the International Conference on Fast Sea Transportation (FAST'09), Athens, Greece, 2009.

[14] M. Altosole, M. Figari, and M. Viviani, "6 DOF Simulation of maneuvering and propulsive performance of water jet propelled Mega Yacht," in Proceedings of the International Conference on Fast Sea Transportation (FAST '09), Athens, Greece, 2009.

[15] A. C. Hochbaum, F. Stern, K. Agdrup et al., "Final report and recommendations to 25th ITTC," in Proceedings of the 25th Information and Telecommunication Technology Center, vol. 1, pp. 143-208, 2008.

[16] R.-Q. Lin and W. Kuang, "Modeling nonlinear roll damping with a self-consistent, strongly nonlinear ship motion model," Journal of Marine Science and Technology, vol. 13, no. 2, pp. 127-137, 2008.

[17] R.-Q. Lin, M. Hughes, and T. Smith, "Prediction of ship steering capabilities with a fully nonlinear ship motion model. Part 1: maneuvering in calm water," Journal of Marine Science and Technology, vol. 15, no. 2, pp. 131-142, 2010.

[18] R.-Q. Lin and W. Kuang, "Modeling the effects of ship appendages on the six-degree of freedom ship motions," in Proceedings of the 2nd International Conference, on Marine Research and Transportation, pp. 25-32, 2007.

[19] R. Q. Lin, W. Kuang, and A. M. Reed, "Numerical modeling of nonlinear interactions between ships and surface gravity waves I: ship waves in calm water," Journal of Ship Research, vol. 49, no. 1, pp. 1-11, 2005.

[20] Lin R.-Q and W. Kuang, "Numerical modeling of nonlinear interactions between ships and surface gravity waves II: ship boundary condition," Journal of Ship Research, vol. 50, no. 2, pp. 181-186, 2006.

[21] S. F. Hoerner, Fluid Dynamic Lift: Theoretical, Experimental and Statistical Information, Hoerner Fluid Dynamics, 1965.

[22] T. C. Shen, "Private communication," 2009.

[23] E. Lewis, Principles of Naval Architecture Second Revision, The Society of Naval Architects and Marine Engineers, New Jersey, NJ, USA, 1998.

[24] R.-Q. Lin and W. Kuang, "A nonlinear method for predicting motions of fast ships," in Proceeding of 10th International Conference on Fast Sea Transportation, Athens, Greece, October 2009. 

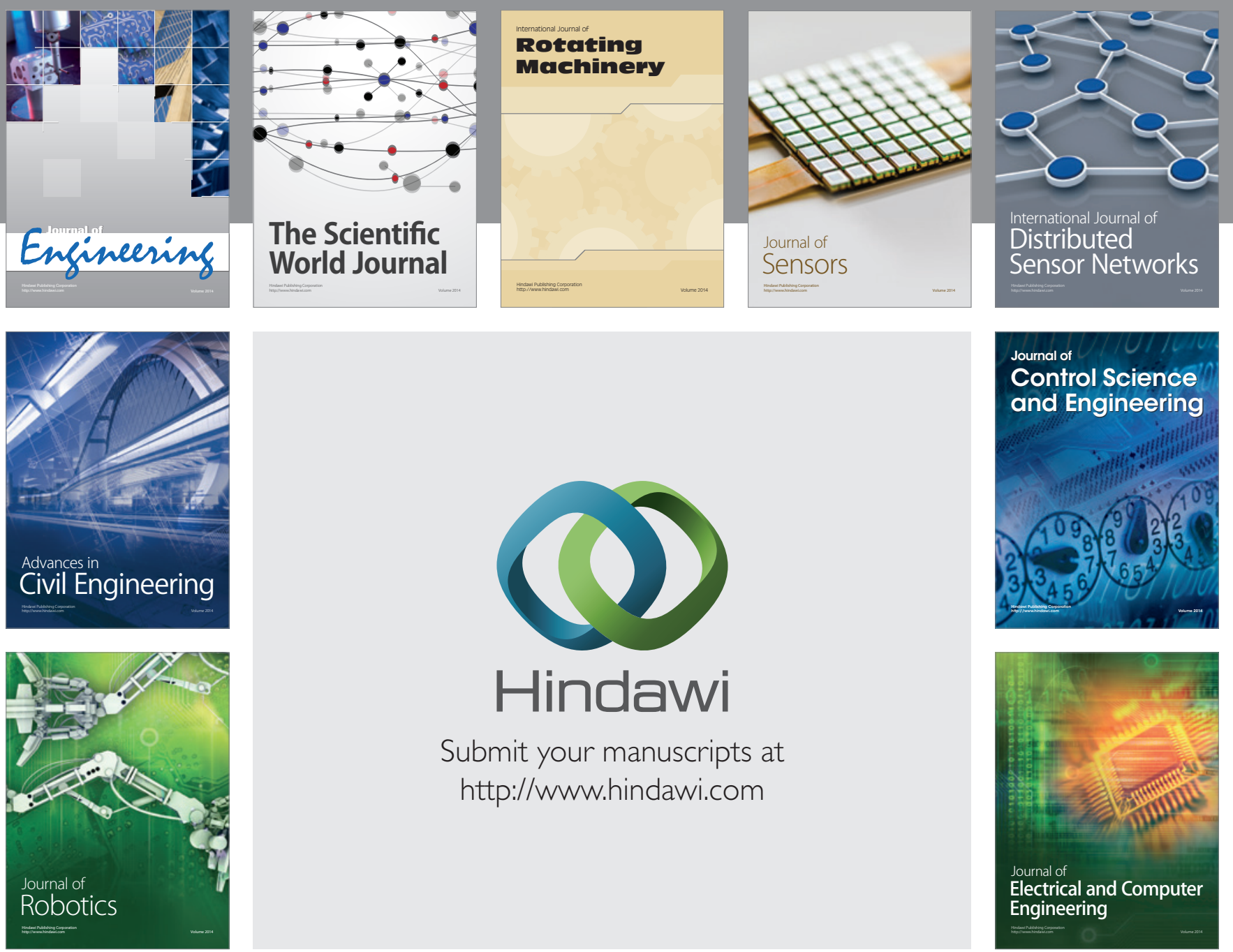

Submit your manuscripts at

http://www.hindawi.com
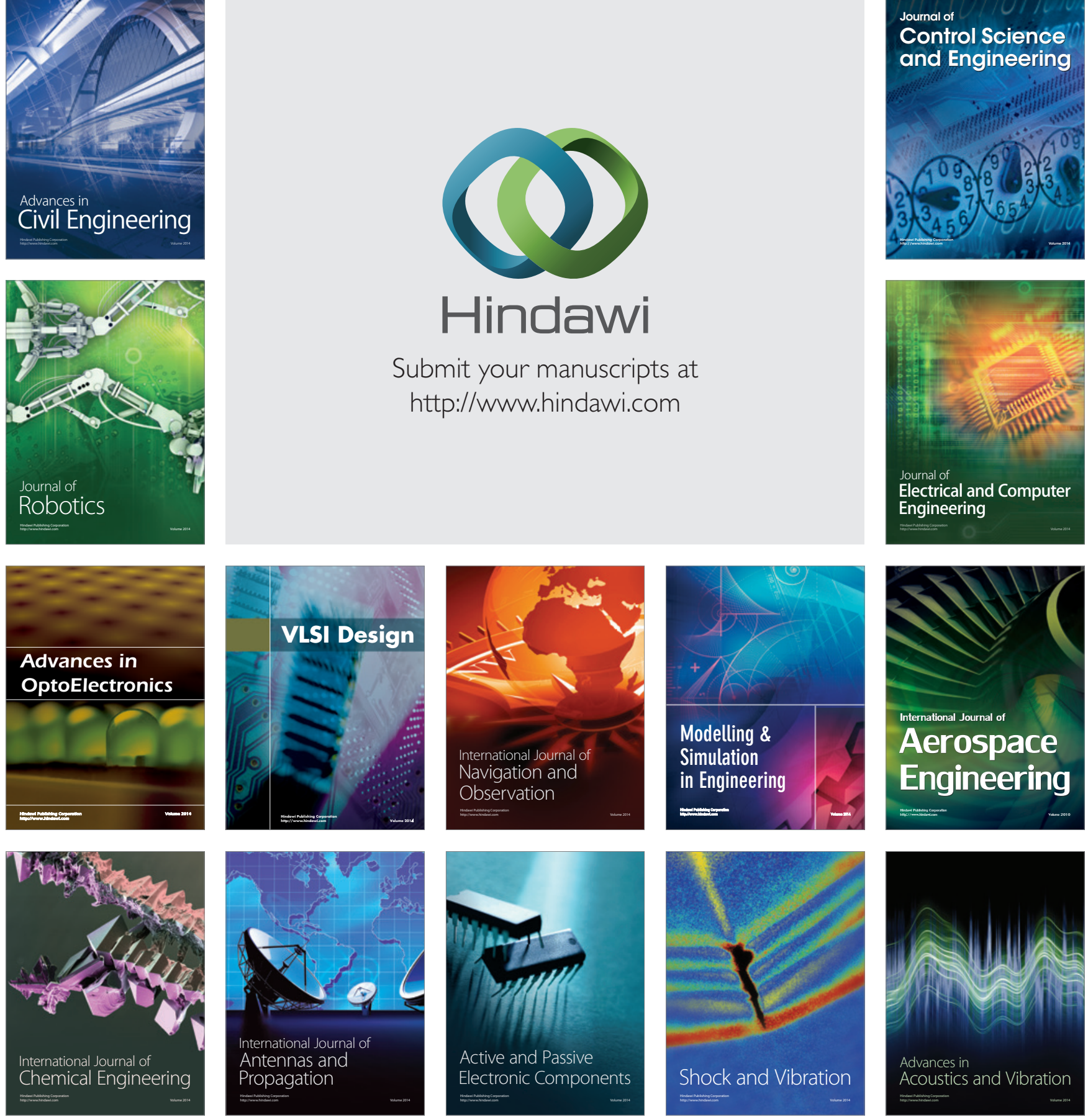